\title{
Nuclear and Particle Physics: Current Issues and Applications (Report on the Nucleus 2020 International Conference)
}

\author{
A. K. Vlasnikov ${ }^{a}$ *, V. I. Zherebchevsky ${ }^{a}$, and T. V. Lazareva ${ }^{a}$ \\ ${ }^{a}$ St. Petersburg State University, St. Petersburg, 199034 Russia \\ *e-mail:a.vlasnikov@spbu.ru \\ Received November 20, 2020; revised December 28, 2020; accepted January 27, 2021
}

\begin{abstract}
The most promising lines of development in nuclear and particle physics are considered. One of these is the synthesis of achievements in nuclear physics and the nano-, bio-, information, cognitive, and social sciences to create technologies similar to nature and improve our understanding of humanity (the NBICS paradigm). The second topic is the growing attention to ultrahigh energies of collision and studying such exotic states of matter as quark-gluon plasma. The reports delivered at the 70th conference on nuclear physics in St. Petersburg provide a wide range of material for discussion.
\end{abstract}

DOI: $10.3103 / \mathrm{S} 1062873821050257$

\section{INTRODUCTION}

The LXX International Conference on Nuclear Physics "Nucleus 2020. Nuclear Physics and Elementary Particle Physics. Nuclear Physics Technologies" was held at St.Petersburg State University October 1217,2020 . This conference is unique not only in the former Soviet Union but worldwide as well. It has been held annually since 1951, hosting leading physicists from many world laboratories. Its predecessors were national conferences on atomic nuclei (held Leningrad in 1933 and Moscow in 1935) and meetings on the physics of atomic nuclei (held in Leningrad in 1938, Kharkov in 1939, and Moscow in 1940). The Second World War interrupted this trend toward holding annual conferences on nuclear physics. Only in February 1951 was the first Conference on Nuclear Physics held in the small hall of the Presidium of the Soviet Academy of Sciences in Moscow [1], where the tradition of regular such meetings was renewed. For many years, the organizing committees of these meetings were headed by Boris Dzhelepov, a corresponding member of the Soviet Academy of Sciences. Due to his authority, leadership, dedication, and enthusiasm, these conferences acquired annual status and were convened at different venues, helping to develop studies of nuclear physics in Russia. Though these conferences were for many years officially referred to as conferences on nuclear spectroscopy, the range of topics grew notably broader, since there were no other annual meetings where nuclear physicists could discuss relevant problems of science. Over time, the title of the conference changed to the National Conference on Nuclear Spectroscopy and Nuclear Structure. It acquired de facto international status in 1960, but con- tinued to be called the National Conference. In the early 2000s, the title was changed to include the word "Nucleus" and the year of the conference. The development of the conference largely repeated that of science in general and nuclear physics in Russia in particular. The number of theses presented in the periods 1951-1970 (Fig. 1a) and 1990-2020 (Fig. 1b) illustrate the development of Soviet and Russian nuclear physics. The rapid increase of the first two decades was replaced by a decline starting in the 1990s. However, growth can be seen over the last five years. It may be uneven, but it gives hope of restoring the role of science in modern society.

The conference of 2020 was unique, and not only because it was the 70th. The conditions under which it was held were most unusual. The Corona virus made the normal conference format impossible. A team capable of conducting a large conference online was gathered in a relatively short period of time. In a situation where many conferences were being cancelled, the joint efforts of St. Petersburg State University, the Kurchatov Institute, the Joint Institute of Nuclear Research, and the conference's organizing committee resulted in the correct strategic and organizational decisions. Reliable channels of communication were established that allowed not only the broadcasting of reports but discussion among the conference's participants as well.

The conference's scientific program covered a broad range of nuclear physics topics: experimental and theoretical investigations of nuclear structure and nuclear reactions, modern methods and technologies in nuclear physics, particle and high-energy physics, neutrino physics, and nuclear astrophysics. Along 


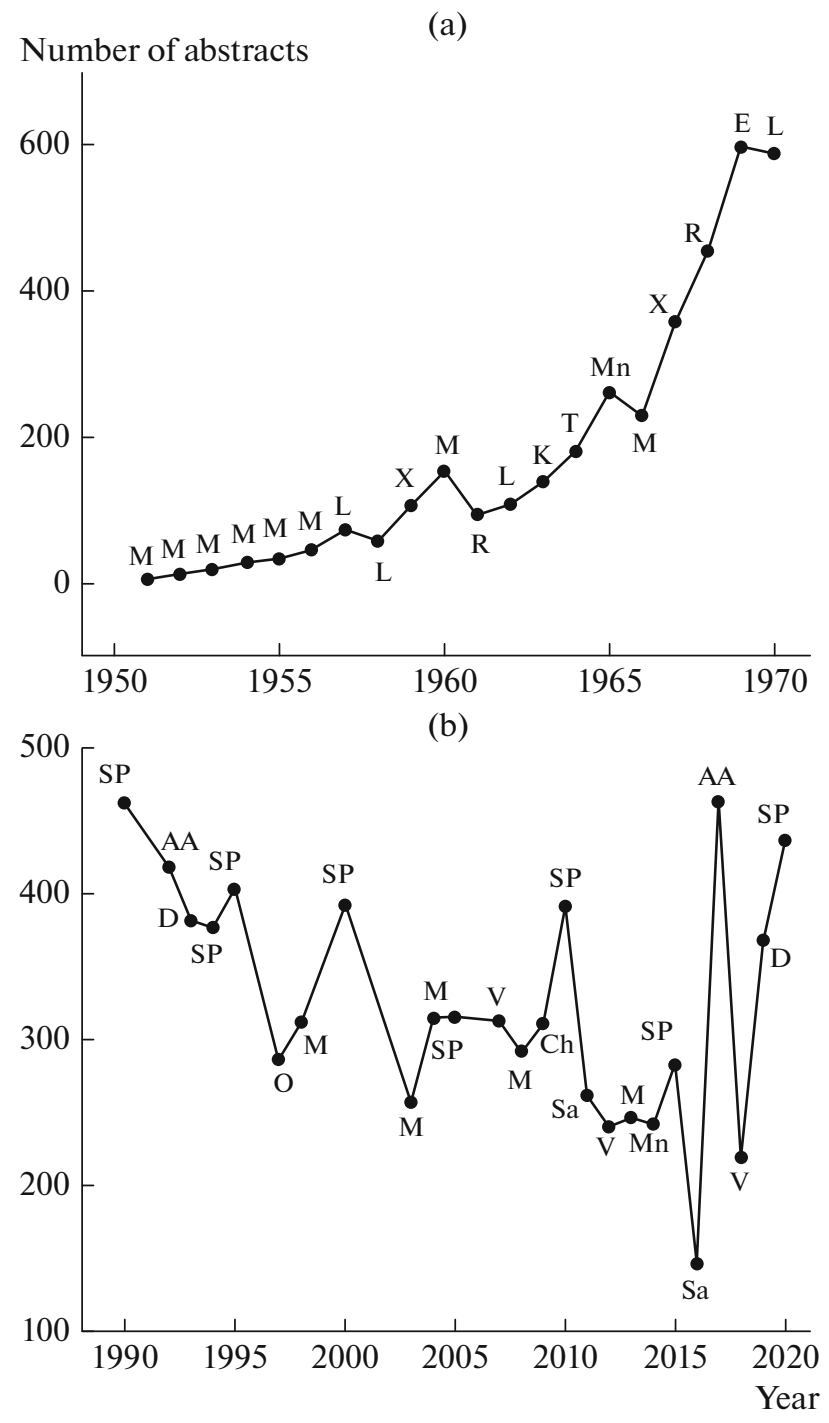

Fig. 1. Change in the number of works included in the programs of annual conferences: (a) 1951-1970 and (b) 1990-2020. Conference venues: Alma-Ata (AA), Voronezh (V), Dubna (D), Erevan (E), Kiev (K), Leningrad (L), Moscow (M), Minsk (Mn), Obninsk (O), Riga (R), Sarov (Sa), St. Petersburg (SP), Tbilisi (T), Kharkov (Kh), and Cheboksary (Ch).

with these traditional topics, the conference's program included reports dealing with synchrotron radiation, neutron physics, and using nuclear physics in studying objects of cultural heritage. Special attention was given to problems encountered in nuclear medicine.

The geography of the conference's participants was also very broad. More than 400 reports were given by particpants from 38 countries (Fig. 2). The total number of registered participants was more than 575. Even though the conference was held online, meaning that participants had to log in during its hours of operation and regardless of time zones (for example, speakers from the University of Illinois in the United States made their presentation at 5:30 in the morning, local time), interest in the reports was very high.

\section{OPENING DAY}

The conference opened on October 12. The participants were greeted warmly by A.E. Blagov (director of the Kurchatov Institute, Moscow), academician V.A. Matveev (director of the Joint Institute for Nuclear Research, Dubna), and Paolo Giubellino (director of GSI Helmholtz Center for Heavy Ion Research, Germany). On behalf of St. Petersburg State University, the conference's participants were greeted by Vice-Rector for Research S.V. Mikushev and conference co-chairman V.I. Zherebchevsky.

On the opening day, a plenary talk titled "Synchrotron Neutron Studies: The Basis for a A Breakthrough in Science and Technology" was delivered by A.E. Blagov (Kurchatov Institute), who reviewed the development of nuclear science and technology in Russia and outlined the current possibilities of synchrotron and neutron studies. It was emphasized that the the Kurchatov Institute is now developing a new interdisciplinary technological system (the NBICS paradigm) that combines nano-, bio-, and information technologies with cognitive and socio-humanitarian knowledge. Nature-like technologies developed in this way will allow us to create biological systems and materials with targeted properties that economize natural resources and energy. An extensive research program that includes experiments with synchrotron and neutron beams must be conducted to achieve these ambitious plans. Blagov noted that the Kurchatov Institute's complex for synchrotron and neutron studies is of the world's few venues where there is a synchrotron (with electron energies of up to $2.5 \mathrm{GeV}$ and 16 experimental stations) and a research reactor on the same territory, allowing a fundamentally new level of basic and applied research to be achieved. The lines of this research include crystallography, materials science, structural chemistry, protein crystallography, molecular biology, medicine, the analysis of organic and hybrid multilayer systems, studies of cognitive processes, and studying objects of cultural heritage. The Kurchatov Institute is a leading center for studies of synchrotron radiation and neutrons in the Russian Federation, so the described program for developing such studies and building the required infrastructure was of special interest. Under the federal program, a network of centers for synchrotron radiation will be created that includes the upgraded source of synchrotron radiation at the Kurchatov Institute, another on Russkiy Island (Vladivostok), and a fourth-generation SKIF installation with an electron energy of $3 \mathrm{GeV}$ (Kol'tsovo, near Novosibirsk). A top-of-the-line fourth-generation source of 


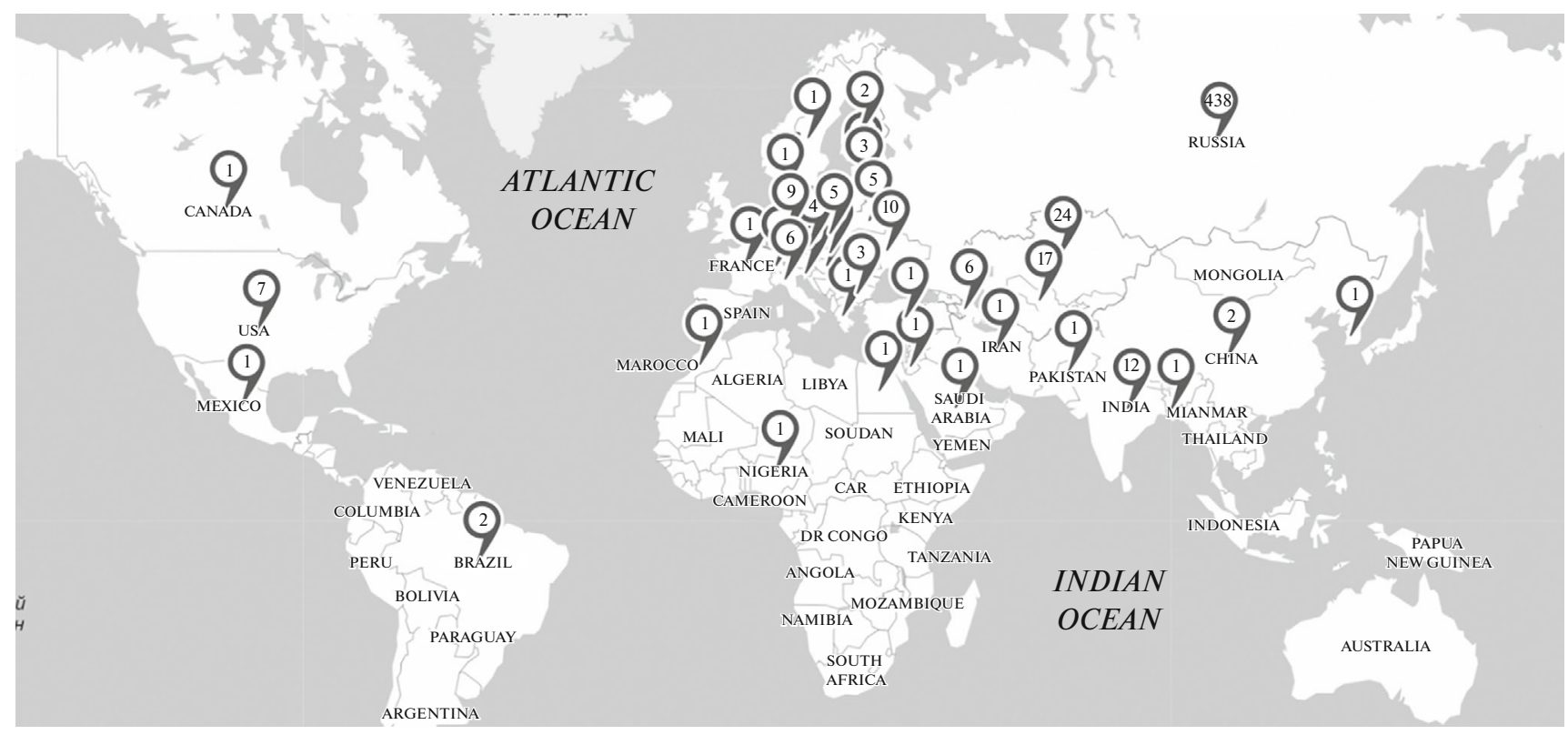

Fig. 2. Conference geography, showing the number of participants per country.

synchrotron radiation with an electron energy of $6 \mathrm{GeV}$ will play a key role in the development of worldclass studies by combining the general infrastructure with a linear accelerator and a free-electron X-ray laser (the SILA project in Protvino, Moscow oblast). The specialized source of synchrotron radiation will have a record low emittance of $90 \mathrm{pm}$ rad, while the femtosecond laser will generate light with a wavelength of $0.1 \mathrm{~nm}$, the level of the world's best installations. These parameters will allow investigations of fast processes and objects the size of atoms.

The plenary report of N.V. Marchenkov, acting director of the Kurchatov Complex of Synchrotron an Neutron Investigations, titled "Kurchatov Complex of Synchrotron and Neutron Research: Current Status and Prospects," allowed the conference's participants to become better acquainted with the research on neutrons and synchrotron radiation now under way on the institute's main campus.

An example of the NBICS paradigm is using means of nuclear physics to study objects of cultural heritage ("Studying Historic Materials by Means of Nuclear Physics at the Kurchatov Institute," presented by E.B. Yatsyshina). Interesting results were yielded by nondestructive tests of materials contained in relic crosses dating back to 10th and 11th centuries, found in Suzdal' Opol'e (a territory in Northeastern Russia heavily populated during the Middle Ages). These crosses were similar to one another but, as neutron tomography showed, contained very different substances (human hair, sheep's wool, and linen and silk fibers). Using computer tomography, a joint research team from the Kurchatov Institute and State Museum of Fine Arts examined ten Egyptian mummies dating back to the 20th century BC. This investigation was complicated by the sarcophagi containing the mummies, which could not be opened.

The investigations revealed the mummification technique, age, gender, diseases, wounds and, in some cases, the cause of death for each mummy. Images of these people that lived 4000 years ago were reproduced using Gerasimov's techniques for reconstructing facial soft tissues. Nuclear physics techniques for investigating artifacts were included in the conference agenda for the first time, drawing considerable attention. This will hopefully continue in future nuclear physics conferences of the series.

The PIK high-flux research reactor (Petersburg Nuclear Physics Institute) is one of the largest megascience facilities operating in the Russian Federation. In his plenary talk titled "International Center for Neutron Research Based on Reactor PIK", Deputy Director V.V. Voronin described current and planned experiments with this Facility. It was noted that the PIK reactor was first mentioned in the literature as early as 1966. Although it was commissioned over half a century ago, this facility still operates more efficiently than most research reactors built recently. Upon the final upgrade of this reseach facility scheduled for 2024, the reactor's power will reach $100 \mathrm{MW}$, and both cold and ultracold neutrons will be available to users. This will allow experimental studies in the fields of condensed-state physics, nanosystems, biology, nuclear and particle physics, and fundamental interactions. 


\section{SECOND DAY}

The second day of the session started with the report "History of One Calendar Date. To the 80th Anniversary of the Discovery of Spontaneous Fission" delivered by S.V. Khlebnikov, director of the Radium Institute Museum (St. Petersburg). Leningrad physicists G.N. Flerov and K.A. Petrzhak, who discovered this phenomenon, built upon significant advances in nuclear physics, radiochemistry, and radiogeology largely achieved at the Radium Institute of the Russian Academy of Sciences. The concept of charged-particle acceleration with the field of a highfrequency alternating current was proposed at the Radium Institute by L.V. Mysovskii, and the first European cyclotron was commissioned there fifteen years later (in 1937). The First National Radioactivity Meeting, the first nuclear physics conference in the Soviet Union, was convened at the Radium Institute in 1932. It is noteworthy that though 1940 is the accepted date of the discovery of spontaneous fission, this phenomenon was first recorded in the report by K.A. Petrzhak (Radium Institute) a year earlier.

Reports on large-scale international experiments in neutrino physics were delivered the same day. In his talk "Status and Prospects of the Jiangmen Underground Neutrino Observatory," Alberto Garfagnini (Italy) represented the JUNO international collaboration, in which physicists from nineteen countries including Russia participate. In the JUNO project, the $700 \mathrm{~m}$ deep experimental hall under construction in China will house the world's largest liquid scintillator detector of antineutrinos: 20000 tons of liquid scintillator will be contained in a spherical vessel with a $30 \mathrm{~m}$ radius, and electron antineutrinos will be detected from the Vavilov-Cherenkov radiation produced by secondary positrons in collisions with protons by 20000 large and 26500 small photomulplier tubes. Two nuclear power plants operating at distances of $\sim 50 \mathrm{~km}$ from the detector will serve as antineutrino sources. Detecting neutrino oscillations will provide clues to the neutrino mass hierarchy and help refine the values of neutrino-mixing parameters. Compared to measurements made with other detectors, the uncertainties on these parameters will be reduced by several times, due to a greater number of statistics. JUNO will also offer a powerful instrument for studies of solar and atmospheric neutrinos, and ones emitted by supernovas and geoneutrinos. The JUNO experimental program also includes seaches for proton decay (predicted by some theoretical schemes beyond the Standard Model) and sterile neutrinos.

The latter were also discussed by A.P. Serebrov (Corresponding Member, Russian Academy of Sciences) in his talk "Observation of Sterile Antineutrino Oscillation in the Neutrino-4 Experiment at SM-3 Reactor" devoted to the revolutionary discovery of a new types of neutrino. Neutrinos ot three flavors corresponding to three generations of lepton are now known to exist: electron, muon, and tau neutrinos. Also hypothesized is the existence of so-called sterile neutrinos, which could be dark matter particles and participate only in gravitational interactions but possibly mix with neutrinos of the three mentioned flavors. Mixing between known and sterile antineutrinos can be studied by measuring the electron-antineutrino flux as a function of the distance from the reactor core. Such measurements made in 2014-2019 in the Neutrino-4 experiment indeed indicate the existence of a sterile neutrino (dubbed neutrino-4), which could also explain results obtained earlier in the LSND and MiniBooNe experiments. Using the Neutrino-4 data, the authors extracted the mass-square difference between the first and fourth neutrino mass eigenstates and the sine of the doubled mixing angle between them. Using data obtained by other experiments and a number of assumptions (including the simplest scheme with a single sterile neutrino, the $3+1$ model), effective neutrino masses were obtained as $m_{\mathrm{ve}}=0.58 \pm 0.09 \mathrm{eV}$, $m_{v \mu}=0.42 \pm 0.24 \mathrm{eV}, m_{v \tau} \leq 0.65 \mathrm{eV}$, and $m_{4}=2.68 \pm$ $0.13 \mathrm{eV}$. The above estimate of the electron-neutrino mass is consistent with corresponding upper limit $m_{v \mathrm{e}}<1.1 \mathrm{eV}$ (at $90 \%$ C.L.) imposed using the tritium beta-decay data of the KATRIN experiment (Germany) and reported by N. Titov (Institute for Nuclear Research, Moscow).

Provided that the lepton number is not conserved and that neutrino is a Majorana particle identical to its antiparticle, the rate of neutrinoless double beta decay $(0 v \beta \beta)$ is also sensitive to the neutrino effective mass. Results from the search for neutrinoless double betadecay in the GERDA experiment (Gran Sasso laboratory, Italy) were reported by F. Salamida. The most stringent lower limit on the half-life of ${ }^{76} \mathrm{Ge} 0 v \beta \beta$ decay was imposed in this experiment: $T_{1 / 2}>1.8 \times 10^{26} \mathrm{yr}$ (90\% C.L.).

Very interesting talks dealing with the physics of ultrahigh-energy cosmic rays were also delivered on the same day. It is well known that the energies of some charged cosmic particles bombarding the Earth's atmosphere exceed by seven-eight orders of magnitude those attained at the highest-energy accelerator constructed so far, the Large Hadron Collider (LHC). These produce showers of secondary particles in the atmosphere that are detected by terrestrial cosmic-ray arrays, particularly by the world's largest observatory, the Pierre Auger in Argentina. We obtain unique astrophysical data by detecting these ultrahigh-energy cosmic rays, along with information on fundamental interactions. Where particle colliders are concerned, we refer the reader to numerous conference reports dealing with the NICA collider under construction at the Joint Institute for Nuclear Research in Dubna. 


\section{THIRD DAY}

Virtually all reports of large international collaborations in the fields of particle physics, relativistic nuclear physics, and high-energy physics were delivered on Wednesday, October 14. The corresponding section of the conference featured the largest number of talks delivered and drew the largest audience, reflecting the worldwide interest in these fields of research. The plenary talks presented results obtained at the Relativistic Heavy-Ion Collider (Brookhaven National Laboratory, US), the Large Hadron Collider (CERN, Geneva), and the CERN proton supersynchrotron. These machines are used in experiments conducted by large international collaborations with considerable Russian participation.

In his report "PHENIX Highlights," Yu. Mitrankov (St. Petersburg Polytechnical University) described studies of quark-gluon plasma (QGP) produced in heavy ion collisions in the PHENIX experiment (RHIC). Compared to the LHC, the RHIC experimental conditions are advantageous in that we can collide heavy ions of different species, thus probing the dependence of QGP formation on the mass numbers of colliding nuclei. PHENIX data were presented on such QGP-sensitive parameters as the anisotropies of charged and neutral secondary hadrons (elliptical and triangular flows), nuclear modification factors reflecting QGP effects on the multiplicity of secondary hadrons, and direct-photon yields. The authors of this report concluded that collisions between light and heavy nuclei result in mini-QGP formation. The data suggest that in all heavy-ion collision systems, direct photons are emitted by sources of the same (albeit not specified) nature, regardless of the energy of collision.

The results from RHIC experiments were also discussed in the report "STAR Recent Results on HeavyIon Collisions" delivered by A.A. Aparin (Joint Institute for Nuclear Research, Dubna). Mapping the phase state of hadronic matter as a function of collision energy and matter density (the so-called phase diagram) is an important task of high-energy physics. Thereby, one can probe the phase transition between the confined and deconfined (or QGP) phases of hadronic matter. In the STAR experiment at the RHIC, the energy range of $\mathrm{AuAu}$ collisions will be extended downward to $\sqrt{s_{N N}}=3 \mathrm{GeV}$, so the boundary between the confined and QGP phases can be investigated. Apart from this future program, A. Aparin reported measurements of the hypertriton-antihypertriton mass difference with which the fundamental CPT symmetry is probed. This proved to be consistent with zero, so no CPT violations were detected.

The results obtained in CERN experiments were discussed in a great many plenary talks. S. Kovalsky (Poland) reported recent studies of strong interactions in the NA61/SHINE fixed-target experiment at the SPS machine, in which the momenta of incident Ar, $\mathrm{Xe}$, and $\mathrm{Pb}$ ions were varied between $13 A$ and
$158 A \mathrm{GeV} / c$. The main aim of this experiment was to study the formation of fireballs (clusters of ultradense, strongly interacting matter) and systematically investigating the hadron gas transition to the QGP phase. Phase diagrams with such variables as temperature, baryon chemical potential, and system size will be plotted for different strongly interacting collision systems. Compared to earlier SPS experiments, greater attention is given in NA61/SHINE to variations of physical characteristics as signals of a phase transition. Variations in the multiplicities of secondary strange and charmed particles are known to signal the onset of QGP formation. As was emphasized by the reporter, the range of collision energies provided by the SPS machine offers a unique opportunity to investigate charmed-particle production in the vicinity of the critical point of the first-order phase transition between the confined and QGP states of hadronic matter.

The current status and upgrade of the ALICE experiment at the LHC were discussed in a number of talks that included four plenary ones. E. Fragiacomo (CERN) reported ALICE data for proton collisions with heavy $\mathrm{Pb}$ and $\mathrm{Xe}$ ions at energies of collision between 1 and $13 \mathrm{TeV}$, shedding light on QGP formation and subsequent decay, and on the planned upgrade of the ALICE detector. The LHC will undergo a substantial upgrade whereby its luminosity will be boosted in two steps (by a factor of ten after 2027). The LHC will resume operation with protons and heavy ions upon completion of the first stage of the upgrade planned for 2021-2022. As reported by W. Trzaska (Finland), the ALICE detector is undergoing a thorough upgrade that will allow this detector of heavy-ion collisions to fully utilize the enhanced LHC luminosity. Upon completion of the detector upgrade in 2022, rare phenomena will be detected with increased precision. These include the emission of heavy-quark hadrons with small transverse momenta sensitive to quark interactions with the medium, and dilepton emissions from QGP that provide insight into the restoration of chiral-symmetry in quark-gluon plasma. The talk "New Inner Tracking System (ITS) for Open Charm Direct Measurements by ALICE at the LHC: Status and Perspectives" delivered by G.A. Feofilov (St. Petersburg State University) was of considerable interest to the audience. Planned upgrades of the detector oriented toward the third and fourth stages of the ALICE experimental program were discussed. Short-lived hadrons containing heavy quarks (such as $D$ mesons) with small transverse momenta will be detected at a much higher frequency of primary collisions using a new internal tracker formed by seven layers of monolithic active pixel sensors manufactured with CMOS technology. The new internal tracker increases the spatial resolution by a factor of three, allows us to detect particles with transverse momenta down to $50 \mathrm{MeV} / c$ (zero for charmed particles), and can perform at a higher parti- 
cle frequencies corresponding to those of primary collisions. In some respects, the new internal tracker of the ALICE detector is superior to those of the ATLAS and CMS detectors also operating at the LHC. Along with probing QGP properties, the ALICE detector can be used to investigate exotic nuclei, as was explained by A. B. Borissov in his talk "Latest Results on (Anti-)Hypernuclei Production at the LHC with ALICE." Apart from elementary particles, lead ion collisions also produce low $A$ nuclei and smaller amounts of hypernuclei that feature a constituent $\Lambda$ hyperon instead of a neutron. We can not only detect hypertritons but also measure their lifetimes, due to the unique virtues of the ALICE detector. The latter proved to be close to the $\Lambda$ lifetime, in agreement with theoretical models predicting that the $\Lambda$ hyperon is weakly bound to constituent nucleons of the nucleus. Antihypertriton production can also be investigated in the ALICE experiment.

That QGP studies at the LHC are not restricted to the ALICE experiment was demonstrated by O. Evdokimova's talk "New Results from Heavy-Ion Studies in the CMS Experiment." Originally, the main thrust of the CMS experiment was particle physics, and particularly those of the Higgs boson. However, the CMS experimental agenda has since been diversified to include studies of heavy-ion collisions (particularly of the azimuthal anisotropy of secondary particles, the production of hadrons containing heavy quarks, and jet quenching). These processes provide clues to QGP formation in heavy-ion collisions. In the latter report, particular attention was given to CMS data on QGP formation in collisions between light nuclei.

\section{FOURTH AND SUBSEQUENT DAYS}

Fundamental problems of low-energy nuclear physics were discussed on the fourth day of the conference. Talks were submitted by veterans who have regularly participated in such conferences for several decades. Spectroscopic factors broadly used in analyzing data on nuclear reactions were critically reviewed by Prof. L.D. Blokhintsev (Moscow State University). It was unexpectedly concluded that spectroscopic factors are unobservable quantities that can be consisitently defined only for certain forms of the nuclear interaction Hamiltonian.

The phenomenon of chaos in quantum-mechanical systems, and in atomic nuclei in particular, was discussed by Prof. V.E. Bunakov (Petersburg Nuclear Physics Institute). How can chaotic motion be defined for quantum processes that involve no particle trajectories? A chaoticity criterium also applicable to quantum systems was proposed: a system can be viewed as chaotic as soon as the number of its integrals of motion (or "good" quantum numbers) is less than the number of degrees of freedom. In counting the number of "good" quantum numbers, we need consider only those that are conserved in the classical limit (in contrast to, e.g., the space parity conserved in strong interactions). In his review, Prof. R.V. Jolos (Joint Institute for Nuclear Research, Dubna) discussed the nuclear phase transitions that occur upon raising the energy of excitation and angular momentum while varying the number of constituent neutrons. The symmetry of the nucleus mean field and the structures of its ground and excited states are thus affected. Such effects as the coexistence of different nuclear shapes, the transition to a state with octupole deformation with increasing angular momentum, and the variation of the nucleus superfluid properties were considered within either the collective and microscopic approach.

JINR measurements of total cross sections of the ${ }^{8} \mathrm{Li}$ and ${ }^{8} \mathrm{He}$ exotic isobar scattering by ${ }^{28} \mathrm{Si},{ }^{59} \mathrm{Co}$, and ${ }^{181} \mathrm{Ta}$ targets as functions of collision energy were reported by V.V. Samarin. Note that the structure of exotic nuclei near drip line consisting of a core and a halo can be efficiently probed by colliding them with stable nuclei, so such experiments attract growing attention. Total cross sections were measured for the first time over a broad energy range of 6-46 MeV per nucleon, and nuclear collisions were detected via prompt neutron and gamma emission. Measurements were compared to theoretical predictions, including ones based on the time-dependent Schrödinger equation, with which the ${ }^{8} \mathrm{Li}$ nucleus was found to consist of a ${ }^{7} \mathrm{Li}$ core (formed by ${ }^{4} \mathrm{He}$ and ${ }^{3} \mathrm{H}$ clusters) and a halo neutron.

In his interesting report "Physical Criteria of Data Reliability and Systematic Uncertainties of Photoneutron Reaction Cross Sections," Prof. V.V. Varlamov (Moscow State University) analyzed the differences between the Saclay and Livermore measurements of the cross sections of photoneutron reactions. In a talk titled "Mechanisms of Multy-Stage Nuclear Decays with Taking into Account Real and Virtual States of Intermediate Nuclei," Prof. S.G. Kadmensky (Voronezh) proposed including the virtual (and real) states of intermediate nuclei in the decay chain of radioactive nuclei. This theoretical framework was then used to describe ternary and quaternary nuclear decays (either spontaneous or induced) as virtual processes. Presentations delivered by E. Litvinova (West Michigan University, United States) and Yu.V. Popov (Moscow State University) were also met with interest.

\section{MEDICAL APPLICATIONS}

The Nucleus 2020 agenda included a broader scope of nuclear physics applications in medical research than those of the previous conferences of the series. European and Russian participants both shared their experience with nuclear physics tecnologies in the therapy and diagnostics of various diseases. The promising modern technology of flash therapy, which 
opens up new possibilities in the treatment of oncological diseases, was described in an interesting report delivered by Prof. M. Dosanjh (CERN). Cancer tumors are briefly exposed to a charged-particle beam and receive a large dose of radiation. The period of irradiation is selected so that the DNA molecules of cancer cells are destroyed, while those of normal cells remain intact. Modern ways of treating oncological diseases with radioactive isotopes of different elements were reviewed by V.I. Zherebchevsky (St. Petersburg State University). These new isotopes find important applications in diagnostics (through positron emission tomography and single-photon emission computerized tomography) and therapy (through radioimmunology). Oncological diseases can be effectively diagnosed and treated with minimum side effects by combining the radioisotope visualization of organs and tissues with radioisotope therapy (an approach referred to as theranostics, or diagnostics-based therapy). The onco-selective preparation administered to the patient contains a radionuclide used originally for diagnostics and then as a means of treatment. Particular attention was given to joint studies and projects with the participation of physicists and engineers from St. Petersburg State University and State Corporation ROSATOM.

\section{CONCLUSIONS}

Because of volume restrictions, not all reports delivered at the conference can be noted in this brief review. For a full list, the reader is referred to Internet site [2] and the list of abstracts published in advance of the conference [3]. The best reports recommended by the conference's program committee will be published in several issues of the Bulletin of the Russian Academy of Sciences: Physics, Physics of Elementary Particles and Atomic Nuclei, and Nuclear Physics and Engineering.

The unique long-term continuity of the series of Russian nuclear physics conferences is explained by the flexibility and broad scope of its scientific program, which adequately reflects the evolution of microworld physics. Compared to the conferences of the initial forty-year period, which dealt largely with low-energy physics, today's emphasis is on highenergy physics and nuclear physics applications in such areas as medicine and studying objects of cultural heritage. Despite the long history of the conference, its organizers are quite young, providing it with new ideas and energy.

\section{ACKNOWLEDGMENTS}

This conference was a result of the collective efforts of many physicists. We thank the participants for sharing their results and ideas. We also thank the conference's audience for their many questions, which could inspire future research leading to new discoveries.

\section{REFERENCES}

1. Izv. Akad. Nauk SSSR, Ser. Fiz., 1953, vol. 16, no. 3, p. 1.

2. LXX Int. Conf. "NUCLEUS 2020: Nuclear Physics and Elementary Particle Physics. Nuclear Physics Technologies," St. Petersburg, 2020. https://indico.cern.ch/event/839985.

3. Book of Abstracts, LXX Int. Conf. "NUCLEUS 2020: Nuclear Physics and Elementary Particle Physics. Nuclear Physics Technologies," St. Petersburg, 2020. https://indico.cern.ch/event/839985/attachments/2116081/ 3590424/book_of_abstracts_Nucleus-2.pdf.

Translated by A. Asratyan 